\title{
Fabrication of octacalcium phosphate foams with suitable mechanical strength for use as a bone substitute based on the setting reaction of acidic calcium phosphate granules
}

\author{
Yuki SUGIURA $^{1, \dagger}$, Asuka OONO ${ }^{1}$ and Yoji MAKITA ${ }^{1}$ \\ ${ }^{1}$ Health and Medical Research Institute, National Institute of Advanced Industrial Science and Technology (AIST), \\ 2217-14 Hayashi-cho, Takamatsu 761-0395, Japan
}

\begin{abstract}
Octacalcium phosphate (OCP) blocks are attractive feedstocks for new biomaterials because of their excellent biocompatibility. Attaching an interconnected porous structure (i.e., a foam) is a good way to use OCP as a bone substitute because this structure allows tissue penetration in the materials. However, the foaming structure shows a lower mechanical strength compared to block-formed materials. In this study, we fabricated OCP foams with enough mechanical strength from calcium dihydrogen phosphate granules of different sizes (100-250, 250$500,500-1000$, and $1000-2000 \mu \mathrm{m})$ through a setting reaction using an acidic phosphate solution and a phaseconversion process. The fabricated OCP foams were found to have high enough mechanical strength $(>0.5 \mathrm{MPa}$ in diametral tensile strength) and porosity $(\sim 70 \%)$. For small granules, a relatively low concentration of acidic phosphate solution was suitable for the setting reaction.

(02020 The Ceramic Society of Japan. All rights reserved.
\end{abstract}

Key-words : Octacalcium phosphate (OCP), Foam, Bone substitute, Phase conversion, Setting reactions

[Received July 2, 2020; Accepted August 12, 2020]

\section{Introduction}

Octacalcium phosphate [OCP: $\mathrm{Ca}_{8}\left(\mathrm{PO}_{4}\right)_{4}\left(\mathrm{HPO}_{4}\right)_{2}$. $\left.5 \mathrm{H}_{2} \mathrm{O}\right]$ is the main component of immature bones and shows excellent biocompatibility when implanted into bone defects. ${ }^{1)-4)}$ Therefore, OCP has attracted attention as a new bone substitute. However, one serious drawback of OCP is its low formability. It can only be fabricated through aqueous-solution-mediated reactions because it decomposes at the required sintering temperatures. ${ }^{1), 5)-7)}$

To use calcium phosphate as a bone substitute, a fabrication process is needed that can produce at least several hundred-micrometer-sized blocks because powder-formed calcium phosphates cause harmful effects such as inflammation. ${ }^{1), 8), 9)}$ In addition, such calcium phosphate blocks must have enough mechanical strength $(>0.5 \mathrm{MPa}$ in diametral tensile strength, DTS) to avoid unexpected fractures. ${ }^{10)-13)}$ Furthermore, attaching an interconnected porous structure (i.e., a foam) to the materials is required because this structure does not only enable penetration into the bone tissue of the materials but also enhances their biocompatibility. ${ }^{11), 14)-16)}$

A dissolution-precipitation reaction using precursor blocks has mainly been employed for the fabrication of thermally unstable calcium phosphates such as carbonate

\footnotetext{
Corresponding author: Y. Sugiura; E-mail: yuki-sugiura@ aist.go.jp
}

apatite and OCP. ${ }^{17)-19)}$ In this procedure, a Ca-based ceramic precursor block is immersed in a suitable solution to stimulatory cause dissolution of the precursor block and precipitation of the desired phases. ${ }^{11), 17), 20)}$ The advantage of this method is that it maintains the shape of the precursor block, which means that blocks of required shapes can be fabricated. ${ }^{12), 21)-23)}$ We previously introduced an OCP foam fabricated from a mixture of calcium sulfate and sodium dihydrogen phosphate. ${ }^{24)}$ Although an in vivo study indicated that the fabricated OCP foam showed an excellent biocompatibility and could be replaced in bones with interior vascular formation, the DTS value of the fabricated OCP was $\sim 0.15 \mathrm{MPa}$, which is much lower than the value required for clinical use. Furthermore, any residual $\mathrm{SO}_{4}$ in OCP foams fabricated from calcium sulfate may be harmful to surrounding tissues.

In further evaluations, we focused on acidic calcium phosphates (i.e., calcium dihydrogen phosphate anhydrate [DCPA: $\mathrm{CaHPO}_{4}$ ], calcium dihydrogen phosphate dihydrate [DCPD: $\mathrm{CaHPO}_{4} \cdot 2 \mathrm{H}_{2} \mathrm{O}$ ], and monocalcium dihydrogen diphosphate monohydrate [MCPM: $\mathrm{Ca}\left(\mathrm{H}_{2} \mathrm{PO}_{4}\right)_{2}$. $\left.\mathrm{H}_{2} \mathrm{O}\right]$ ) as precursors of OCP. ${ }^{25)-28)}$ These materials and their formation process had been applied as bone cements, normally, liquid forms acidic phosphate containing $\mathrm{Ca}$ solutions called as brushite cement. Their crystal-growth velocities were relatively high, and therefore, when these crystals were formed in the cracks and veins of granules, an interlocking process between them enabled binding the 
target materials as cementing materials. ${ }^{30), 31)}$ In fact, various calcium ceramics could be bonded using acidic calcium phosphates. ${ }^{32)-34)}$

This process and the properties of the materials have advantages for fabricating interconnected porous structures because this binding is not uniform but partial, so many pores could be formed. ${ }^{32), 34)}$ If the pore sizes and structures are optimized employing such a setting reaction, this technique could be useful for fabricating foam-like structures of OCP materials. It might be considered that the key of this process is a balance between the dissolution of the base calcium phosphate and the precipitation of the acidic calcium phosphate crystals. This process may control the composition (i.e., the $\mathrm{Ca}$ and $\mathrm{PO}_{4}$ concentration) of the cement materials. Therefore, in this study, we investigated how the liquid-cement composition affects both the mechanical properties of OCP foams and their intermediate materials.

\section{Experimental method}

\subsection{Fabrication of the precursor ceramic foam}

In this study, an acidic calcium phosphate foam was formed in two steps including the fabrication of acidic calcium phosphate granules and their setting reaction. ${ }^{10), 30), 33), 34)}$ Except for $\beta$-tricalcium phosphate $[\beta$ TCP: $\left.\mathrm{Ca}_{3}\left(\mathrm{PO}_{4}\right)_{2}\right]$, all the reagents were purchased from FUJIFILM Wako Pure Chem Inc., Japan. $\beta$-TCP was purchased from Taihei Chemical Industrial Inc., Japan. Acidic calcium phosphate granules were fabricated as analogs of the dental brushite cement setting reaction. ${ }^{10), 30), 33), 34)}$ MCPM and $\beta$-TCP were mixed at a molar ratio of 1:1 using an agate mortar and pestle. Then, $\sim 1 \mathrm{~g}$ of the mixture was put into a rotary pan-type granulator (PZ-01R, As One Co., Japan) with stirring at $40 \mathrm{rpm}$. Approximately $1.0 \mathrm{~mL}$ of distilled water was added as a spray to obtain granules of acidic calcium phosphate. The set mixture was stirred continuously for $10 \mathrm{~min}$ and then placed in a dry oven at $40^{\circ} \mathrm{C}$ overnight.

The dried set mixture was separated, using an automatic sieve, into five granule sizes, specifically, <100, 100-250, 250-500, 500-1000, and 1000-2000 $\mathrm{m}$. All the sizes (except $<100 \mu \mathrm{m})$ were used for further treatment. These size ranges are hereafter simply described as "100-250," "250-500", and so on.

An acidic phosphate solution for the setting reaction was prepared from $1 \mathrm{~mol} / \mathrm{L} \mathrm{H}_{3} \mathrm{PO}_{4}$, adding MCPM until it was saturated. The sieved granules were placed into silicon rubber molds of $\varphi=6 \times 3 \mathrm{~mm}$. Then, the MCPMsaturated $\mathrm{H}_{3} \mathrm{PO}_{4}$ (acidic phosphate) solution $(50 \mu \mathrm{L})$ was dropped onto the sieved granules, which were immediately packed for the setting reaction. After a few minutes, they were placed into a dry oven at $60^{\circ} \mathrm{C}$ for several hours to remove any moisture. The set granules were stored at $40^{\circ} \mathrm{C}$ for further reactions. The concentration of MCPMsaturated $\mathrm{H}_{3} \mathrm{PO}_{4}$ was changed between 0.1 and $1.0 \mathrm{~mol} / \mathrm{L}$ to evaluate the feasibility of the acidic phosphate solution treatment. The concentrations of the acidic phosphate solutions are described here in terms of the $\mathrm{H}_{3} \mathrm{PO}_{4}$ concentration.

The setting behavior of the granules was described as one of three possibilities: (1) did not set, (2) set with suitable form, or (3) set without pores. In these cases, the condition "did not set" was defined for granules that - after treatment- could not be picked up using tweezers. The "set without pores" condition was defined for the case when the granules had shrunk significantly after the treatment, reaching a diameter below $4.8 \mathrm{~mm}$ (i.e., a shrinking ratio greater than $20 \%$ ). Between these two conditions, the granules were said to have "set with suitable form."

\subsection{Fabrication of an OCP foam through a dissolution-precipitation reaction from set acidic calcium phosphate granules}

Ten samples of the set granules (approximately $1 \mathrm{~g}$ ) were immersed into $20 \mathrm{~mL}$ of $1 \mathrm{~mol} / \mathrm{L} \mathrm{Na}_{2} \mathrm{HPO}_{4}$ at $70^{\circ} \mathrm{C}$ for three days. The treated set granules were then washed with distilled water several times and placed in a dry oven at $40{ }^{\circ} \mathrm{C}$ to remove residual moisture.

\subsection{Characterization}

The sample morphology was studied by field emission scanning electron microscopy (SEM; JSM-6700F, JEOL Co., Japan) at an accelerating voltage of $3 \mathrm{kV}$ after coating with osmium. The compositions of the samples were analyzed by X-ray diffraction (XRD; MiniFlex600, Rigaku Co., Japan) at $40 \mathrm{kV}$ and $15 \mathrm{~mA}$, through crushing processes using an agate mortar and pestles. The diffraction angle was continuously scanned in a $2 \theta$ range of $3-70^{\circ}$. The chemical-bonding structure of the samples was determined by Fourier-transform infrared spectroscopy (Nicolet Nexus 670, ThermoFisher Scientific Co., USA) using a triglycine sulfate detector (64 scans, $2 \mathrm{~cm}^{-1}$ resolution) with a GeSe attenuated-total-reflection prism. The measurements were obtained in air.

The mechanical strengths of the samples were evaluated in terms of their DTS. After measuring the diameters and heights of the specimens using a micrometer (MDC25MU, Mitutoyo Co., Japan), they were crushed using a universal testing machine (AGS-J, Shimadzu Co., Japan) at a constant cross-head speed of $1 \mathrm{~mm} / \mathrm{min}$. The average values and standard deviations of the DTS $(\sigma)$ were calculated from the results of the breaking strength $(P)$ of five specimens $(N=5)$ using the following equation:

$$
\sigma=2 P / \pi d l
$$

Here, $d$ is the diameter of the specimen and $l$ is its length.

The macroporosity $\left(P_{\mathrm{m}}\right)$ of the samples was calculated by the bulk density method, based on the following equation:

$$
P_{\mathrm{m}}=\left(2.67 \times 10^{3}-w / \pi d l\right) \times 100
$$

Here, $w$ is the weight of the sample and $2.67 \times 10^{3}$ $\mathrm{kg} / \mathrm{m}^{3}$ is the density of solid OCP. ${ }^{32)}$ The average values and standard deviations of the porosity were calculated 
using the results from five specimens $(N=5)$. An analysis of the internal morphology was performed using a quantitative three-dimensional evaluation program included in the microcomputed tomography system (microCT; Skyscan 1075 KHS, Kontich, Belgium) at a source voltage of $69 \mathrm{kV}$ and a source current of $149 \mu \mathrm{A}$ with a $0.5 \mathrm{~mm} \mathrm{Al}$ filter. The voxel resolution was $9 \mu \mathrm{m}$.

\section{Results and discussion}

\subsection{Evaluation of the DCPA granules and} their setting reaction through an acidic calcium phosphate solution treatment

First, we characterized the fabricated granules via setting reactions. Figure 1 shows the XRD patterns (a) and SEM micrographs $(b-e)$ of the set granules. All the sieved granules were characterized as DCPA, whereas the mixture of MCPM and $\beta$-TCP was partially converted into DCPD. The SEM micrographs indicated that the granules were rectangular and consisted of sub-micrometer-sized particles. The aspect ratio of the granules was around 1-3.
Then, we evaluated the relationship between the concentration of acidic phosphate solutions and the properties of the foam-like structures made from the DCPA granules through the setting reaction [Fig. 2(a)]. As noted, there were three categories that were used to denote the behavior of the setting reaction. These are shown in the figure as: (b) did not set, (c) set with interconnected pores (suitable form), and (d) set without interconnected pores. For the setting reaction in the case of small granules, a relatively low concentration of acidic phosphate solution was suitable.

Figure 3 shows typical photographs (a) and microCT images $(b, c)$ of the set DCPA granules, which maintained the whole shapes of the molds. In addition, the microCT images of the samples showed that the set DCPA granules had interconnected porous inner structures.

Figure 4 shows SEM images of the set DCPA granules. Their surface is covered by sub-micrometer block-like crystals, similar to the surface of the DCPA granules before the treatment.


Fig. 1. (a) XRD patterns for $\beta$-TCP, MCPM, a mixture of the two, and for each size range of the granules. SEM images of: (b) $100-250 \mu \mathrm{m}$ granules, (c) $250-500 \mu \mathrm{m}$ granules, (d) $500-1000 \mu \mathrm{m}$ granules, and (e) 1000 $2000 \mu \mathrm{m}$ granules.

(a)



Fig. 2. (a) The setting condition of the DCPA granules as a function of the acidic phosphate concentration. Schematic images of granules that (b) did not set, (c) set with pores (suitable form), and (d) set without pores. 



Fig. 3. Photographs (a) and microCT whole images (b) and slice images of the set DCPA granules.
The set granules were further characterized by XRD. Figure 5 shows the XRD patterns of set DCPA granules made using an acidic phosphate solution treatment. The set DCPA granules mainly consisted of DCPA and DCPD with MCPM. Considering the peaks of MCPM $d_{(010)}$ (at $\sim 7.5^{\circ}$ ) and DCPA $d_{(120)}\left(\right.$ at $\sim 30.3^{\circ}$ ) peaks relative intensities in XRD patterns of the DCPA granules after treatment and setting situation, the amount of MCPM in the set granules increased with the concentration of the acidic phosphate solution. ${ }^{35), 36)}$ In any cases, MCPM formed by acidic phosphate solution treatment onto DCPA granules banded DCPA granules each other.

The mechanical strength of the set DCPA granules was described later using the results obtained after the treatments.

\subsection{Evaluation of the phase conversion proc- ess of set DCPA granules.}

Figure 6 shows the final $\mathrm{pH}$ values of the treated solutions. Although they slightly decreased with increasing acidic phosphate concentration, all the final $\mathrm{pH}$ values of


Fig. 4. SEM micrographs of the set DCPA granules before immersion, showing (a, b) 100-250, (c, d) 250-500, (e, f) 500-1000, and (g, h) 1000-2000. Top: low magnification; bottom: high magnification.

(a) $100-250$



(b) $250-500$



(c) $500-1000$



(d) $1000-2000$



Fig. 5. XRD patterns of DCPA granules of different sizes treated with various concentrations of acidic phosphate solution, showing (a) 100-250, (b) 250-500, (c) 500-1000, and (d) 1000-2000. 
the treated solutions were approximately neutral, which is suitable for OCP formation. ${ }^{36)-38)}$

Figure 7 shows typical photographs (a) and microCT images $(b, c)$ of the set DCPA granules after immersion. Their overall shape and inner structures were maintained after immersion in $1 \mathrm{~mol} / \mathrm{L} \mathrm{NaH}_{2} \mathrm{PO}_{4}$. Comparing the samples before and after immersion shows that the process caused a slight coloration, especially transpiration changing.

Figure 8 shows the XRD patterns of the set DCPA granules after immersion in $1 \mathrm{~mol} / \mathrm{L} \quad \mathrm{NaH}_{2} \mathrm{PO}_{4}$. The patterns indicate that the material turned into monophasic OCP. Based on these results, we refer to the set DCPA granules after immersion as OCP foam.

We then examined how the immersion process affected the fine structure of the set DCPA granules after conversion into OCP foam. Figure 9 shows SEM images of the OCP foams. It can be seen that the surface of the OCP foams consists of plate-like crystals with sizes in the micrometer range which is a typical OCP crystal morphology.



Fig. 6. Final $\mathrm{pH}$ values of a treated $1 \mathrm{~mol} / \mathrm{L} \mathrm{Na}_{2} \mathrm{HPO}_{4}$ solution as a function of the concentration of treated acidic phosphate solution. $\bigcirc: 100-250, \bigcirc: 250-500, \diamond: 500-1000$, and $\diamond$ : $1000-2000$.

(a) $100-250$



(b) $250-500$



The mechanical strength (Fig. 10) and porosity (Fig. 11) of the OCP foams were determined to evaluate the feasibility of using them as bone substitutes. In addition, the mechanical strength and porosity of the set DCPA granules were determined for comparison. In the case of the treatment with $0.2 \mathrm{~mol} / \mathrm{L}$ acidic phosphate, the set DCPA granules collapsed during immersion in $1.0 \mathrm{~mol} / \mathrm{L} \mathrm{Na}_{2} \mathrm{HPO}_{4}$. At relatively low acidic phosphate concentrations and small granule sizes, the mechanical strength of the OCP foam remained almost constant with increasing acidic phosphate concentration. However, at a relatively high acidic phosphate concentration, the mechanical strength of the set DCPA granules and the OCP foams showed opposite trends, even though the calcium phosphate foam was converted from the set DCPA granules. Importantly, for 100250, 250-500, 500-1000, and 1000-2000 $\mu \mathrm{m}$ granules, the OCP foams were mechanically strong enough $(>0.5 \mathrm{MPa}$


Fig. 7. Photographs (a) and microCT whole images (b) and slice images (c) of the set DCPA granules immersed in $1 \mathrm{~mol} / \mathrm{L}$ $\mathrm{Na}_{2} \mathrm{HPO}_{4}$ at $70^{\circ} \mathrm{C}$ for three days.

Fig. 8. XRD patterns of the set DCPA granules immersed in $1 \mathrm{~mol} / \mathrm{L} \mathrm{Na} \mathrm{HPO}_{4}$ at $70{ }^{\circ} \mathrm{C}$ for three days, showing (a) 100-250, (b) 250-500, (c) 500-1000, and (d) 1000-2000. 

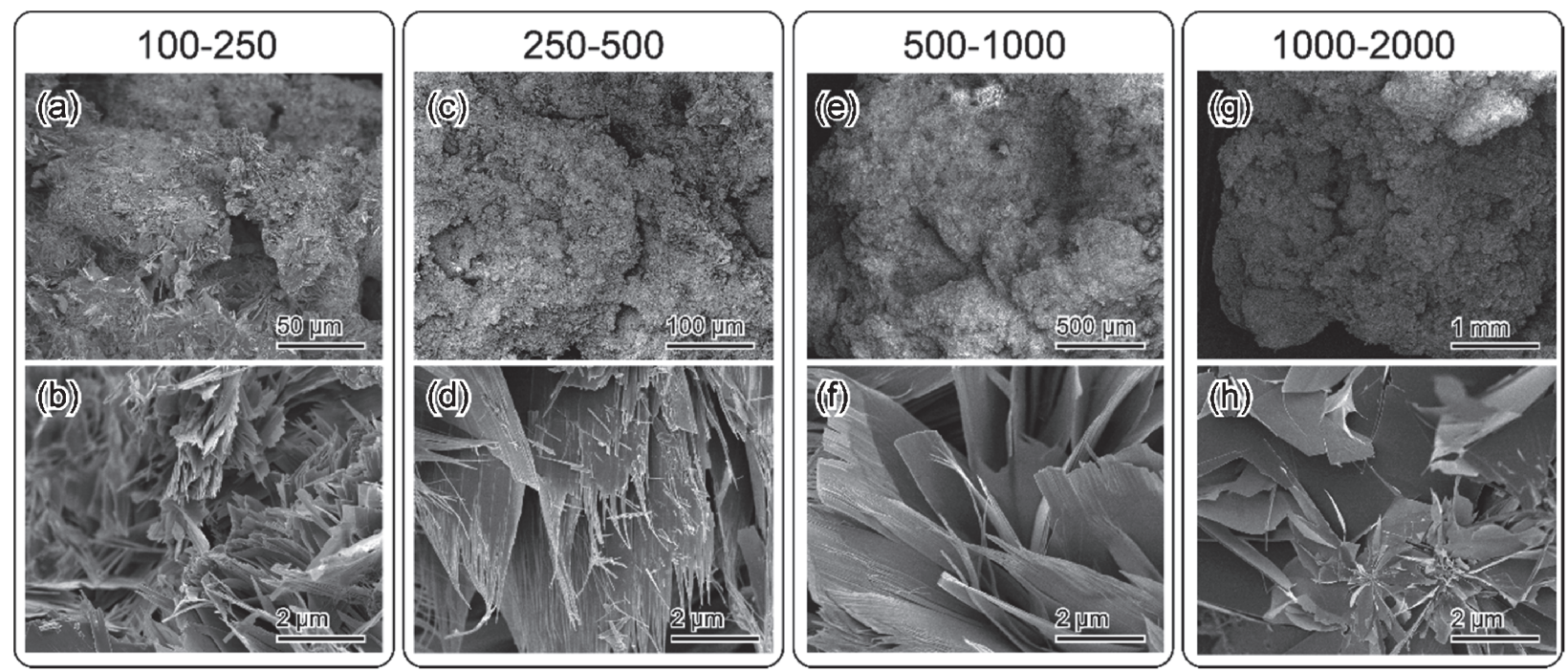

Fig. 9. SEM micrographs of OCP foams, showing (a, b) 100-250, (c, d) 250-500, (e, f) 500-1000, and (g, h) 1000-2000. Top: low magnification; bottom: high magnification.


Fig. 10. DTS values for (a) the set DCPA granules and (b) the OCP foam. Red: 100-250; blue: 250-500; green: 500-1000; purple: $1000-2000$.

in DTS) for use as bone substitutes. There were no significant differences in the porosities of the OCP foams calculated by the bulk density method; they were all around $70 \%$ (Fig. 11). Indeed, in the SEM images, the values obtained for each sample seemed to be much higher than



Fig. 11. Porosity of the OCP foams calculated by the bulk density method. Red: 100-250; blue: 250-500; green: 500-1000; purple: $1000-2000$.

those determined by simple observation. Therefore, we considered that many microscopic pores also formed in the fabricated OCP foams.

In this study, several chemical reactions played important roles in the setting process. For DCPA granules, precursor materials for OCP foams, fabrication, below reaction through moisture mediating let MCPM and $\beta$-TCP to convert completely to DCPA, as shown in Eq. (3):10),31)

$$
\begin{aligned}
& \mathrm{Ca}\left(\mathrm{H}_{2} \mathrm{PO}_{4}\right)_{2} \cdot \mathrm{H}_{2} \mathrm{O}: \mathrm{MCPM}+\mathrm{Ca}_{3}\left(\mathrm{PO}_{4}\right)_{2}: \beta-\mathrm{TCP} \\
& \quad \rightarrow 4 \mathrm{CaHPO}_{4}: \mathrm{DCPA}+3 \mathrm{H}_{2} \mathrm{O}
\end{aligned}
$$

Then, during the setting reactions of the DCPA granules, several processes caused the precipitation and interlocking of the DCPD crystals. When an acidic calcium phosphate solution was dropped onto the DCPA granules, these dissolved according to the following chemical reaction, Eq. (4):

$$
\mathrm{CaHPO}_{4}+\mathrm{H}^{+} \rightarrow \mathrm{Ca}^{2+}+\mathrm{H}_{2} \mathrm{PO}_{4}^{-}
$$


Note that when DCPA is dissolved in an acidic solution, the phosphate molecules adsorbed $\mathrm{H}^{+}$along to $\mathrm{PO}_{4}$ chemical equilibrium. Then, the $\mathrm{pH}$ of the solution increases as the dissolution of DCPA proceeds. Upon increasing the $\mathrm{pH}$ from acidic conditions $(\mathrm{pH} \sim 1.0)$, the solubilities of calcium phosphates such as DCPD and MCPM decrease. Therefore, these phases precipitate from the solutions, as shown in Eqs. (5) and (6):

$$
\begin{aligned}
& \mathrm{Ca}^{2+}+\mathrm{H}_{2} \mathrm{PO}_{4}{ }^{-}+2 \mathrm{H}_{2} \mathrm{O} \\
& \quad \rightarrow \mathrm{CaHPO}_{4} \cdot 2 \mathrm{H}_{2} \mathrm{O}: \mathrm{DCPD}+2 \mathrm{H}^{+} \\
& \mathrm{Ca}^{2+}+\mathrm{H}_{2} \mathrm{PO}_{4}{ }^{-}+\mathrm{H}_{2} \mathrm{O} \\
& \quad \rightarrow \mathrm{Ca}\left(\mathrm{H}_{2} \mathrm{PO}_{4}\right)_{2} \cdot \mathrm{H}_{2} \mathrm{O}: \mathrm{MCPM}
\end{aligned}
$$

When these crystals precipitated, their crystal-growth ratios were high; therefore, the crystals were interlocked with each other during the setting reactions.

The obtained results clearly show that OCP foams with enough mechanical strength for use as bone substitutes can be fabricated from DCPA granules through a setting reaction and a phase-conversion process, except for granules with sizes of $1000-2000 \mu \mathrm{m}$ treated with $0.9 \mathrm{~mol} / \mathrm{L}$ acidic phosphate solution. When DCPA granules are treated with $\sim 0.5 \mathrm{~mol} / \mathrm{L}$ acidic phosphate solution, they set in a form that is suitable for OCP foam fabrication because the set DCPA granules, their intermediate materials, became to set with interconnected pores (suitable form) as optimizing acidic calcium phosphate formation during setting reactions. Therefore, it was concluded that $\sim 0.5 \mathrm{~mol} / \mathrm{L}$ acidic phosphate solution was optimal concentration for DCPA granules setting reactions.

MCPM is one of the precursors of OCP, and there is a pseudomorphic transition between MCPM and OCP formed from MCPM crystals under weak-acidic-to-basic conditions. ${ }^{37), 39)}$ Under these conditions, the MCPM crystals interlock with each other. However, an excessive acidic treatment dissolves the DCPA granules and causes shrinking of the compact structures. Therefore, in this reaction system, controlling MCPM formation during the setting reaction is a key factor for obtaining an OCP foam with both suitable form and high enough mechanical strength.

\section{Conclusion}

In this study, OCP foams with high mechanical strength were fabricated from DCPA granules by a setting reaction and a phase-conversion process. In the case of 100-250, 250-500, 500-1000, and 1000-2000 $\mathrm{mm}$ granules, we obtained OCP foams with enough strength $(>0.5 \mathrm{MPa}$ in DTS) to be used as bone substitutes. In the case of largediameter granules, the mechanical strengths of the set DCPA granules and the resulting OCP foams showed an inverse relationship. For setting reactions using small granules, a relatively low concentration of acidic phosphate solution was suitable.

Acknowledgements We thank to Prof. K. Ishikawa and Dr. K. Hayashi, Kyushu University, Japan, for microCT measurement. This study is financially supported by the priority issue of the Health Research Institute, National Institute of Advanced Industrial Science and Technology (AIST), and KAKENHI for Young Researcher, JP19K19081.

\section{Reference}

1) O. Suzuki, Acta Biomater., 6, 3379-3387 (2010).

2) H. Newesely, Mh. Chem., 91, 1020-1023 (1960).

3) F. Barrere, C. M. van der Valk, R. A. Dalmeijer, C. A. van Blitterswijk, K. de Groot and P. Layrolle, J. Biomed. Mater. Res. A, 64, 378-387 (2003).

4) S. V. Euw, Y. Wang, G. Laurent, C. Drouet, F. Babonneau, N. Nassif and T. Azaïs, Sci. Rep., 9, 8456-8466 (2019).

5) R. Z. LeGeros, Calcified Tissue Int., 37, 194-197 (1985).

6) E. Boanini, M. Gazzano, K. Rubini and A. Bigi, Cryst. Growth Des., 10, 3612-3617 (2010).

7) N. Ito, M. Kamitakahara, S. Murakami, N. Watanabe and K. Ioku, J. Ceram. Soc. Jpn., 118, 762-766 (2010).

8) F. Loi, L. A. Córdova, J. Pajarinen, T. Lin, Z. Yao and S. B. Goodman, Bone, 86, 119-130 (2016).

9) S. Narayan, B. Pazar, H.-K. Ea, L. Kolly, N. Bagnoud, V. Chobaz, F. Liote, T. Vogl, D. Holzinger, A. K.-L. So and N. Busso, Arthritis Rheum., 63, 422-433 (2011).

10) K. Tsuru, A. Yoshimoto, M. Kanazawa, Y. Sugiura, Y. Nakashima and K. Ishikawa, Mater., 10, 374-383 (2017).

11) K. Ishikawa, J. Ceram. Soc. Jpn., 127, 595-601 (2019).

12) S. Karashima, A. Takeuchi, S. Matsuya, K. Udoh, K. Koyano and K. Ishikawa, J. Biomed. Mater. Res. A, 88, 628-633 (2009).

13) C. J. Anker, Clin. Orthop. Relat. R., 434, 251-257 (2005).

14) D. Y. Shin, M.-H. Kang, I.-G. Kang, H.-E. Kim and S.-H. Jeong, J. Biomater. Appl., 0, 1-11 (2018).

15) K. Ishikawa, T. S. Putri, A. Tsuchiya, K. Tanaka and K. Tsuru, J. Biomed. Mater. Res. A, 106, 797-804 (2018).

16) K. Ishikawa, T. I. Arifta, K. Hayashi and K. Tsuru, J. Biomed. Mater. Res. B, 107, 269-277 (2019).

17) K. Ishikawa, Mater., 3, 1138-1155 (2010).

18) Y. Sugiura, M. L. Munar and K. Ishikawa, J. Mater. Sci. Mater. Med., 29, 151-158 (2018).

19) G. Tripathi, Y. Sugiura, A. Kareiva, E. Garskaite, K. Tsuru and K. Ishikawa, J. Biomater. Appl., 33, 259-270 (2018).

20) Y. Sugiura, J. Jpn. Assoc. Cryst. Growth, 47 (2020). [in Japanese].

21) N. Koga, K. Ishikawa, K. Tsuru and I. Takahashi, Ceram. Int., 42, 7912-7917 (2016).

22) Y. Ayukawa, Y. Suzuki, Y. Takamori, I. Atsuta, H. Nakamura, T. Sawase, K. Koyano, Y. Hara, K. Ishikawa and K. Tsuru, Bio. Med. Res. Int., 10, 579541 (2015).

23) S. Nomura, K. Tsuru, S. Matsuya, I. Takahashi and K. Ishikawa, Dent. Mater. J., 33, 166-172 (2014).

24) Y. Sugiura and K. Ishikawa, Mater. Lett., 239, 143-146 (2019).

25) Y. Sugiura and Y. Makita, Cryst. Growth Des., 18, 6165-6171 (2018).

26) Y. Sugiura and Y. Makita, Chem. Lett., 47, 1371-1374 (2018).

27) Y. Sugiura, Y. Saito, T. Endo and Y. Makita, Cryst. Growth Des., 19, 4162-4171 (2019).

28) E. Davies, M. J. Duer, S. E. Ashbrook and J. M. Griffin, 
J. Am. Chem. Soc., 134, 12508-12515 (2012).

29) W. E. Brown, Nature, 196, 1048-1050 (1962).

30) L. M. Grover, J. C. Knowles, G. J. P. Fleming and J. E. Barralet, Biomater., 24, 4133-4141 (2003).

31) F. Theiss, D. Apelt, B. Brand, A. Kutter, K. Zlinszky, M. Bohner, S. Matter, C. Frei, J. A. Auer and B. von Rechenberg, Biomater., 26, 4383-4394 (2005).

32) P. T. Kien, K. Ishikawa and K. Tsuru, Ceram. Int., 41, 13525-13531 (2015).

33) Eddy, A. Tsuchiya, K. Tsuru and K. Ishikawa, J. Biomater. Appl., 33, 630-636 (2018).

34) Y. Sugiura, K. Tsuru and K. Ishikawa, J. Mater. Sci.
Mater. Med., 28, 122-128 (2017).

35) G. MacLennan and C. A. Beevers, Acta Crystallogr., 8, 187-190 (1956).

36) L. Wang and G. H. Nancollas, Chem. Rev., 108, 46284669 (2008).

37) Y. Sugiura and Y. Makita, Dalton T., 48, 1386-1391 (2019).

38) G. Vereecke and J. Lemaitre, J. Cryst. Growth, 104, 820-832 (1990).

39) Y. Sugiura, K. Tsuru and K. Ishikawa, J. Ceram. Soc. Jpn., 124, 827-832 (2016). 